\title{
Altersgerechtes Wohnen im Quartier
}

\section{Das Beispiel Margaretenau Regensburg}

\author{
Sonja Haug ${ }^{1}$ (D) $\cdot$ Miriam Vetter $^{1}$ \\ Eingegangen: 29. März 2020 / Überarbeitet: 6. Oktober 2020 / Angenommen: 7. Oktober 2020 / Online publiziert: 28. Oktober 2020 \\ (c) Der/die Autor(en) 2020, korrigierte Publikation 2020
}

\section{Zusammenfassung}

Im Alter nimmt das Quartier aufgrund geringerer Aktionsradien an Bedeutung zu. Der Beitrag befasst sich mit der Frage, welche Besonderheiten sich bei Senioren-Haushalten im Hinblick auf Wohnen und soziale Teilhabe zeigen. Aus dem Projekt Energetische Modernisierung des genossenschaftlichen Wohnquartiers Margaretenau in Regensburg (MAGGIE) werden Ergebnisse einer schriftlichen Haushaltsbefragung $(N=195)$ in der Wohngenossenschaft Margaretenau Regensburg dargestellt. Hierbei wird auf die aktuelle und zukünftige Wohnsituation, den Veränderungsbedarf, den Wunsch nach Gemeinschaftsangeboten und die soziale Einbettung eingegangen. Es zeigt sich eine geringe Veränderungsbereitschaft und ein überdurchschnittlich hohes Maß an lokalem Sozialkapital. Am Ende werden Schlussfolgerungen für das Sanierungsund Quartiersmanagement gezogen.

Schlüsselwörter Senioren · Wohnsituation · Quartiersgestaltung · Sozialraum · Lokales Sozialkapital

\section{Age-appropriate living in the neighborhood}

A case study: Margaretenau in Regensburg

\begin{abstract}
In old age, the neighborhood increases in importance because of moving in smaller circles. The article deals with the question of what special features are evident in senior households in terms of housing and social participation. The results of a written household survey $(N=195)$ in the Margaretenau residential cooperative in Regensburg are presented from the project Energetic Modernization of the Cooperative Residential Neighborhood Margaretenau in Regensburg (MAGGIE). The current and future housing situation, the need for change, and the desire for community opportunities and social integration are analyzed. Little willingness to change and an above-average level of local social capital are shown. At the end, conclusions are drawn with regard to renovation and neighborhood management.
\end{abstract}

Keywords Seniors $\cdot$ Living situation $\cdot$ Neighborhood design $\cdot$ Social space $\cdot$ Local social capital

\section{Einleitung}

Prof. Dr. Sonja Haug

sonja.haug@oth-regensburg.de

Miriam Vetter

vetter.miriam@outlook.de

1 Institut für Sozialforschung und Technikfolgenabschätzung, Ostbayerische Technische Hochschule Regensburg,

Seybothstr. 2, 93053 Regensburg, Deutschland
Es ist eine Gnade, in der Margaretenau wohnen zu dürfen (Quelle: MAGGIE Haushaltsbefragung 2019, Interview Nr. 187).

Besonders im Alter wird im Vergleich zu jüngeren, örtlich unabhängigeren Menschen das Quartier bedeutender (Saup 1993). Ein Grund liegt darin, dass durch körperliche Einschränkungen, fehlende finanzielle Mittel und durch ge- 


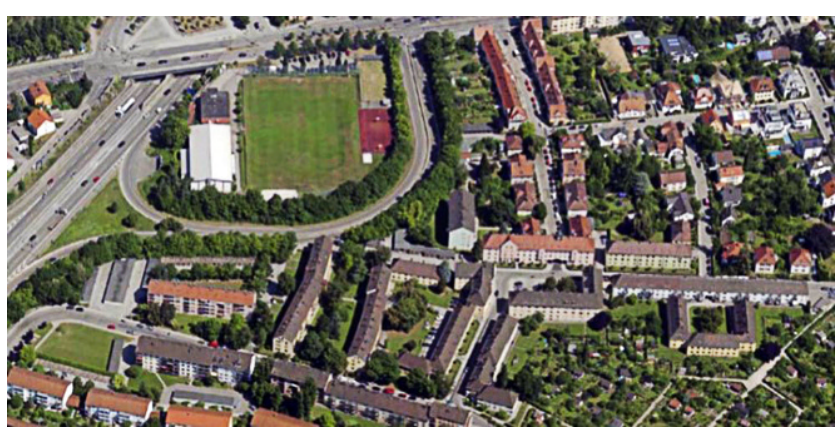

Abb. 1 Schrägaufnahme Luftbild Baugenossenschaft Margaretenau Regensburg 2013. (Quelle: Abteilung Vermessung und Kartographie, Stadt Regensburg, Urheber Foto: AEROWEST GmbH, Dortmund)

ringe Aktionsradien das nähere, räumliche Umfeld seltener verlassener werden kann (Eichler und Holz 2014). Insofern wird auch bei kleinräumigen Sanierungen oder Quartiersneugestaltungen zunehmend eine altersgerechte Perspektive eingenommen. Exemplarisch kann hier auf die Studie von Opitz und Pfaffenbach verwiesen werden, die die Lebensqualität der Bewohnerinnen und Bewohner im ländlichen Raum sozialräumlich analysiert haben (2018). Durch Quartiersgestaltungen und Sanierungsmaßnahmen wird der lokale Nahraum an die Bedürfnisse älterer Menschen angepasst, um ihnen so einen längeren Verbleib in ihrer gewohnten Umgebung zu ermöglichen (Kremer-Preiß 2013).

Der Beitrag befasst sich am Beispiel der Margaretenau Regensburg mit der Frage, wie ältere Menschen im Wohnquartier sozial eingebettet sind und welche Bedürfnisse und Wünsche sie im Hinblick auf eine Gebäude- und Quartierssanierung haben. Die Baugenossenschaft Margaretenau liegt im Westenviertel von Regensburg (Abb. 1). Sie wurde 1918 als Arbeitersiedlung nach dem Gartenstadtmodell gegründet und nach Margarete Clementine Maria von Thurn \& Taxis benannt (Baugenossenschaft Margaretenau 2018).

Ziel des interdisziplinären Forschungsprojekts MAGGIE ist ein Lösungsentwurf für die energetische Gebäudesanierung, der in der Lindenstraße erprobt wird (Abb. 2 und 3). Darauf basierend wird ein Sanierungskonzept für das gesamte Wohnquartier erstellt.

Innovativ ist die Verbindung unterschiedlicher Fachdisziplinen wie Human- und Stadtgeographie, Sozialwissenschaft, Architektur, Bau- und Energietechnik. Im Folgenden werden Ergebnisse der sozialwissenschaftlichen Begleitstudie vorgestellt. Diese Sozialstudie orientiert sich inhaltlich und methodisch an der Studie RENARHIS (Haug und Vernim 2016). Ziel des folgenden Beitrags ist es, Besonderheiten von Seniorenhaushalten im Hinblick auf Wohnen und soziale Teilhabe aufzuzeigen und Implikationen für die Quartiersentwicklung abzuleiten.

Alle Haushalte der Baugenossenschaft Margaretenau wurden im Januar 2019 mit schriftlichen, standardisierten
Fragebögen befragt (Grundgesamt $=344$ Haushalte, Rücklauf $N=195,57 \%$ ). Inhalte waren aktuelles und zukünftiges Wohnen, Einstellungen zur energetischen Sanierung, Mobilitätsverhalten und gesellschaftliche Teilhabe im Quartier. Neben der Haushaltsbefragung wurden amtliche Daten zur Soziodemografie analysiert.

\section{Altersgerechte Quartiersgestaltung}

Der Begriff „altersgerechte Quartiersgestaltung“ zielt auf die seniorenspezifische Anpassung der Wohnung, des Gebäudes, des Wohnumfelds sowie des Quartiers ab (Gädker et al. 2012, S. 2). ,[E]ine ,altersgerechte Wohnung “ umfasst nicht nur eine weitgehend barrierefreie/-reduzierte Wohnung, sondern auch ein barrierefreies/-reduziertes Wohnumfeld, die ortsnahe Verfügbarkeit wesentlicher Infrastruktureinrichtungen sowie soziale und pflegerische Unterstützungsangebote" (Bundesministerium für Verkehr, Bau und Stadtentwicklung BMVBS 2011, S. 25). Zu den Mindestanforderungen für barrierefreies/barrierereduziertes Wohnen gehören nicht mehr als 3 Stufen zum Wohnungseingang, keine Stufen innerhalb der Wohnung, ausreichend Bewegungsflächen und Türbreiten im Sanitärbereich und Vorhandensein einer bodengleichen Dusche (Bundesministerium für Verkehr, Bau und Stadtentwicklung BMVBS 2011, S. 25). Ein altersgerechter Umbau trägt dazu bei, trotz altersbedingter und gesundheitlicher Einschränkungen in der Wohnung verbleiben zu können (KfW Bankengruppe 2014).

\section{Soziodemografie der Quartiersbevölkerung der Margaretenau Regensburg}

Die folgenden Auswertungen basieren auf einer Lieferung von unveröffentlichten Aggregatdaten des Amtes für Stadtentwicklung der Stadt Regensburg, die im Rahmen des Projekts MAGGIE mit EXCEL selbst ausgewertet wurden (Stichtag 31.12.2017). Hierbei wurden jeweils die Daten für die gesamte Stadt der Blockgruppe Margaretenau gegenübergestellt. Insgesamt leben nach in Regensburg 166.467 Einwohner (Stadt Regensburg 2019, S. 69) und in der Blockgruppe Margaretenau 1184 Personen. In der Margaretenau gibt es im Gegensatz zum gesamten Stadtgebiet kein natürliches Bevölkerungswachstum. Die Geburtenrate entspricht der Sterberate. Die Zuzugsrate liegt in Regensburg bei 17,65 pro 1000 Einwohner, in der Margaretenau nur bei 0,13 . In der Margaretenau ist ein geringer Wanderungssaldo und geringes Wanderungsvolumen festzustellen. Sowohl im Stadtgebiet als auch in der Blockgruppe sind die meisten Bewohnenden ledig, wobei in der Margaretenau etwas häufiger Verheiratete, Geschiedene oder Verwitwete 
Abb. 2 Margaretenau, Ausschnitt der Stadtgrundkarte Regensburg. (Quelle: Abteilung Vermessung und Kartographie, Stadt Regensburg, Bearbeitung Luxgreen)

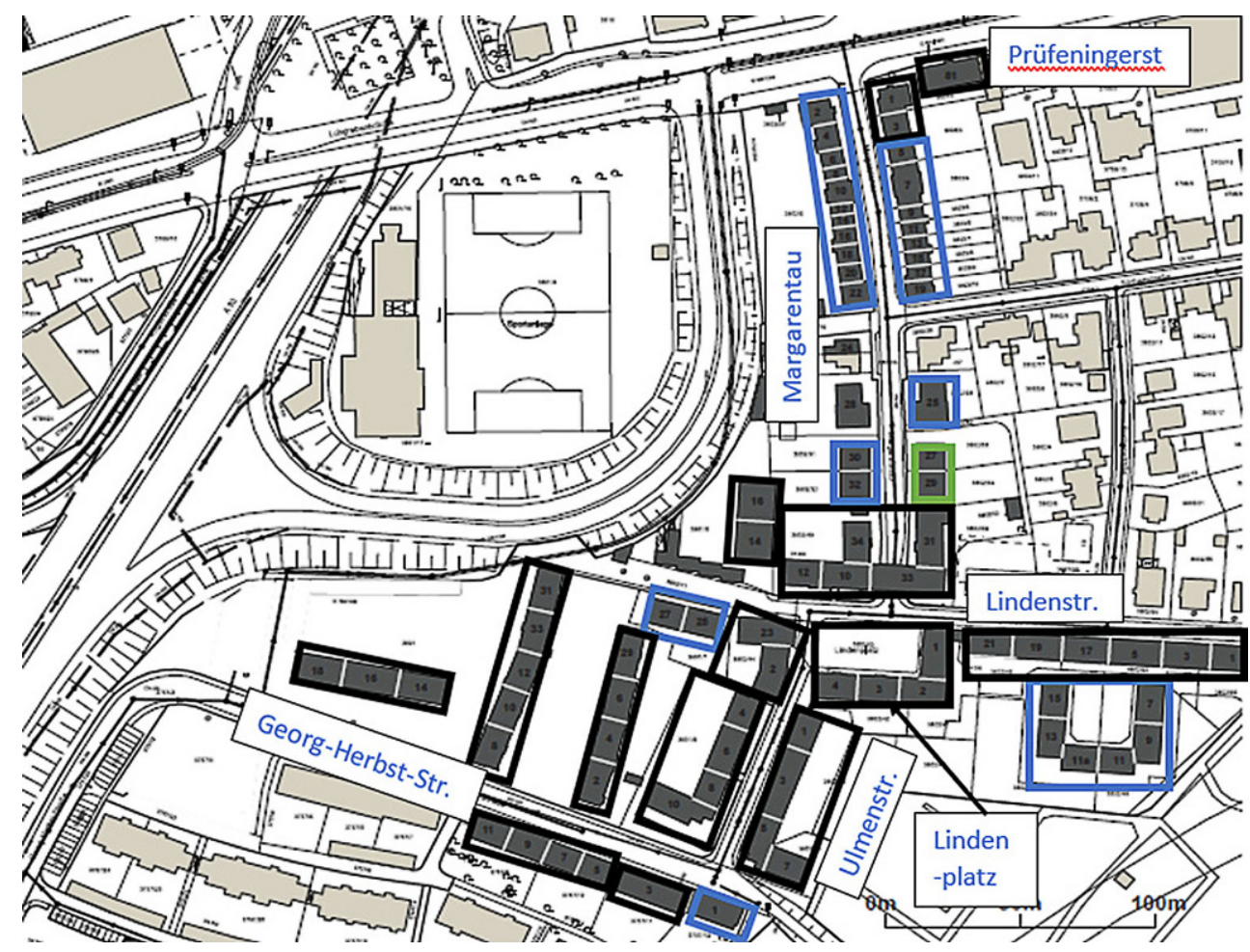

Abb. 3 Gebäude Margaretenau Regensburg. (Foto: Annika Zeitler, OTH Regensburg)

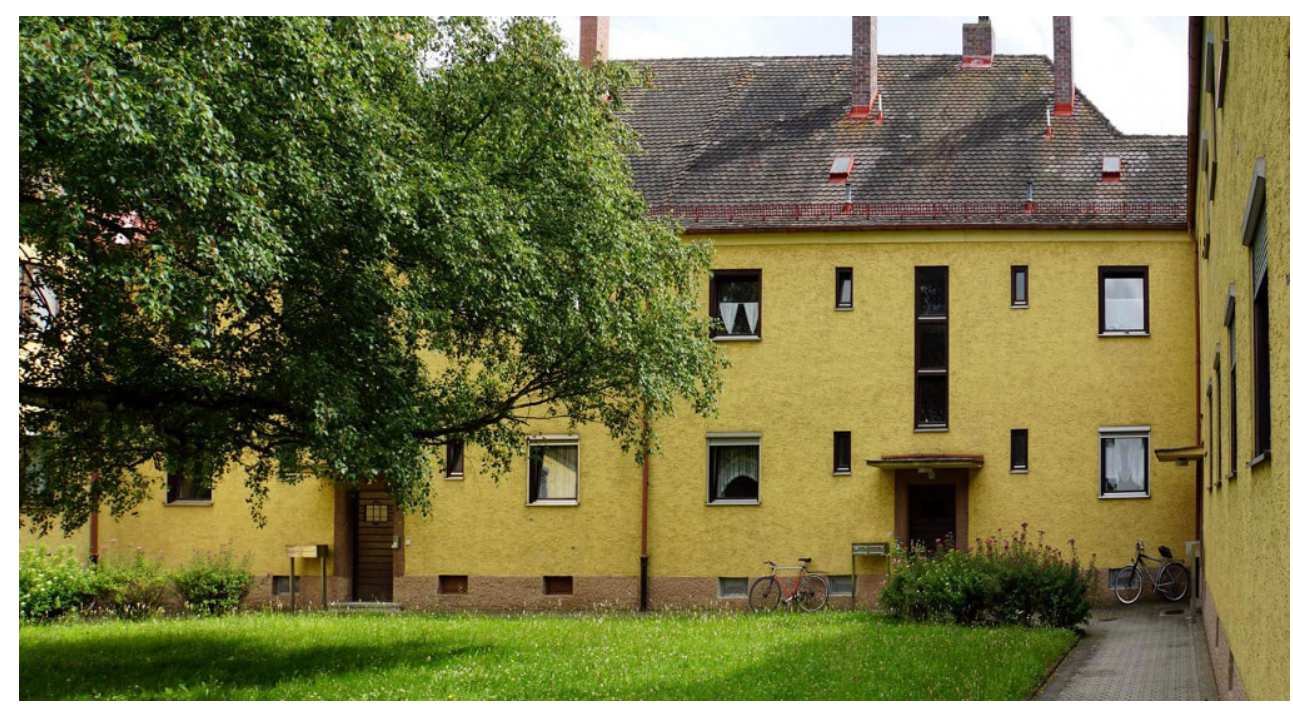

leben. Mehr als die Hälfte leben in Einpersonenhaushalte, im Stadtgebiet 56\% und in der Margaretenau 59\%.

Ein leichter Frauenüberhang mit 54,1\% weiblicher Bewohnerinnen erklärt sich durch einen Altersstruktureffekt und die höhere Lebenserwartung von Frauen. Überdurchschnittliches Alter ist ein wesentliches Charakteristikum von Genossenschaftsmitgliedern (Theurl et al. 2013). Dementsprechend sind in der Blockgruppe Margaretenau die Altersgruppen der über 65-Jährigen und auch der 45- bis 64Jährigen stark vertreten. Sie sind in der Befragung auch überrepräsentiert (Abb. 4).

\section{Veränderungsabsichten im Alter}

Die Haushaltsmitglieder der 195 teilnehmenden Haushalte wurden zusammengefasst (Summe 310 Personen). Für die Analyse wurde zwischen jüngeren Haushalten und Seniorenhaushalten mit mindestens einer 65-jährigen Person (37\% der Haushalte) unterschieden. Seniorenhaushalte sind überwiegend Einpersonenhaushalte. Sie leben in signifikant kleineren Haushalten (M 1,44; SD 0,91) als Nichtseniorenhaushalte (M 1,81; SD 0,53, T-Test $p<0,000)$. Die durchschnittliche Wohndauer in der Margaretenau ist mit 19 Jahren sehr lang (M 18,99; SD 17,10). Seniorenhaushalte le- 
Abb. 4 Altersverteilung. (Datenquellen: Datenabfrage beim Amt für Stadtentwicklung der Stadt Regensburg für Stadt Regensburg und Blockgruppe Margaretenau; Eigene Datenerhebung MAGGIE Haushaltsbefragung 2019, eigene Auswertung)

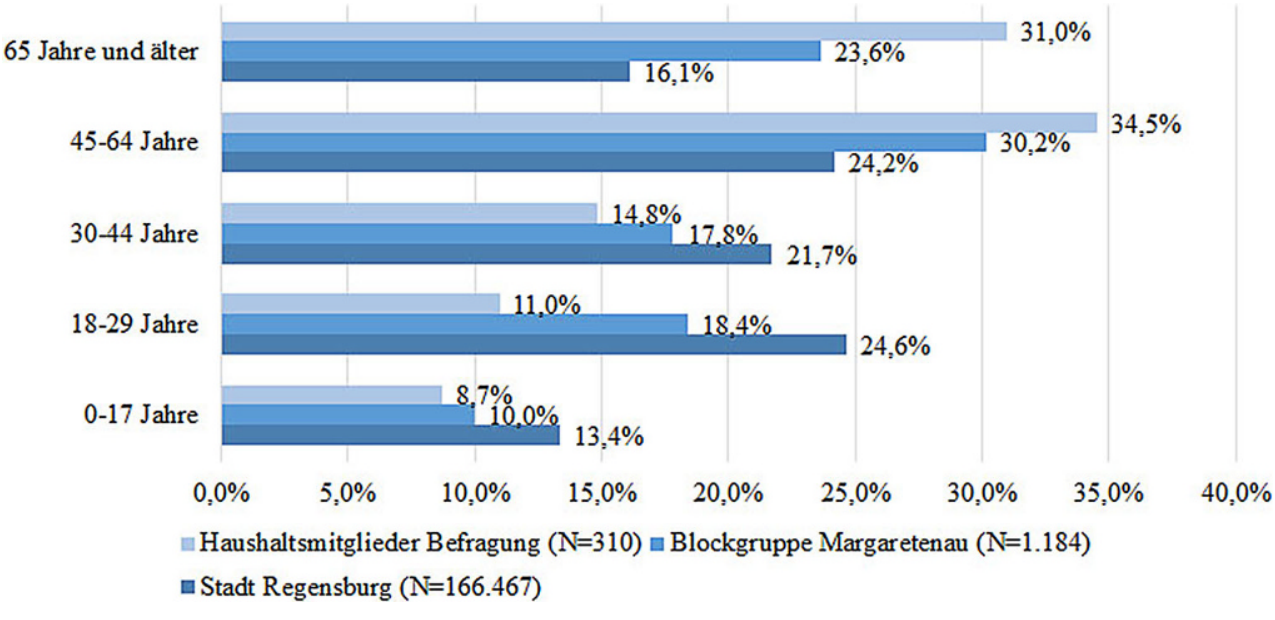

ben durchschnittlich 28 Jahren in der aktuellen Wohnung (M 27,97; SD 19,29), jüngere Haushalte 14 Jahre (M 13,72; SD 13,14, T-Test $p<0,000)$. Insgesamt $80 \%$ wollen dauerhaft bzw. auch im Alter in ihrer Wohnung bleiben, 89,3\% in der Margaretenau leben. Nur 2,8\% der Seniorenhaushalte wollen sich verändern, wohingegen ein Fünftel der Nichtseniorenhaushalte $(23,7 \%)$ über Veränderungsabsichten nachdenkt $\left(\chi^{2}\right.$-Test $\left.p<0,01\right)$. Demzufolge ist ein viel geringerer Anteil der Senioren zum Wohnortwechsel bereit als die umzugsbereiten mover bei Teti et al. mit $29,1 \%$ (2014, S. 324).

Ein Viertel der Haushalte wünscht sich einen Umzug in eine sanierte, $17,8 \%$ in eine barrierefreie und $14,7 \%$ der Befragten einen Umzug in eine größere Wohnung (Tab. 1). Eine Verkleinerung der Wohnsituation ist sehr selten gewünscht, sehr selten ist auch ein Auszug von Haushaltsmitgliedern absehbar. Ein Auszug ist bei Nichtseniorenhaushalten häufiger zu erwarten $\left(\chi^{2}\right.$-Test $\left.p=0,01\right)$. Signifikant häufiger wünschen jüngere Haushalte eine Vergrößerung der Wohnsituation $\left(\chi^{2}\right.$-Test $\left.p<0,000\right)$ und einen Umzug in eine sanierte Wohnung $\left(\chi^{2}\right.$-Test $\left.p<0,000\right)$. Unerwartet ist, dass Seniorenhaushalte etwas seltener an einer barrierefreien Wohnung interessiert sind als Nichtseniorenhaushalte, wobei der Unterschied nicht signifikant ist. Dies ist plausibel, da auch Familien mit Kleinkindern von barrierearmen Wohnungen profitieren. Dass Seniorenhaushalte relativ wenig Bedarf äußern, könnte mit ihrer Mietkostensensitivität oder ihrer Zufriedenheit mit der Wohnung zusammenhängen. Seniorenhaushalte, die einen Umzug in eine barrierearme Wohnung innerhalb der Genossenschaft in Erwägung ziehen, wünschen vor allem ein barrierefreies Bad, wohingegen Barrieren bei Türschwellen oder beim Hauseingang für jüngere Haushalte relevanter sind.

Die Mehrheit ist mit der Wohnausstattung zufrieden (Skala 1: sehr zufrieden, 2: zufrieden, 3: mittel, 4: unzufrieden, 5: sehr unzufrieden. M 2,23; SD 0,95). Der Mittelwert für die Zufriedenheit mit der Miethöhe ist noch höher, er beträgt 1,73 (SD 0,82). Die durchschnittliche Wohnungsmiete lag in der Margaretenau im Jahr 2018 bei $5,16 € / \mathrm{m}^{2}$, in der Stadt Regensburg bei $8,96 € / \mathrm{m}^{2}$ (Stadt Regensburg 2018, S. 5). Die Zufriedenheit für Heizund Stromkosten ist dagegen etwas geringer (Heizkosten M 2,39; SD 0,96; Stromkosten M 2,43, SD 0,91). Letztere deuten auf den Bedarf der energetischen Sanierung hin und auf überdurchschnittlich hohe Energiekosten, die durch die Sanierungsmaßnahmen reduziert werden sollen.

\section{Soziale Teilhabe und genossenschaftliches Zusammenleben}

Das Untersuchungsgebiet ist geprägt von einem dichten Nachbarschaftsgefüge. Die Mehrheit von $92,1 \%$ ist sehr zufrieden oder zufrieden mit dem genossenschaftlichen $\mathrm{Zu}$ sammenleben. Wöchentlichen Kontakt zur Nachbarschaft pflegt knapp die Hälfte der Haushalte von 48,2\%. Verstärkt wird dies durch verwandtschaftliche Beziehungen. Jeder Zweite hat eine verwandte Person im näheren Umfeld. Seniorenhaushalte zeigen sich zufriedener und weisen mehr nachbarschaftliche Interaktionen auf. Umgekehrt wünscht sich ein Viertel der jüngeren Haushalte signifikant häufiger mehr Kontakt zu ihrer Nachbarschaft.

Drei Viertel der Befragten sehen einen Bedarf für Gemeinschaftseinrichtungen (Tab. 2). An erster Stelle interessieren sich 46,2\% der Haushalte für einen Quartiersladen. Auch Bedarfe für einen Außensitzplatz (40,0\%) oder einen ambulanten Pflegedienst $(26,7 \%)$ werden genannt. Einer Begegnungsstätte und einer Freizeitstätte stehen nur etwa ein Fünftel der Befragten offen gegenüber. Gemeinschaftsaktivitäten im kulturellen oder sozialen Bereich werden von etwa einem Zehntel gewünscht. Nichtseniorenhaushalte legen signifikant mehr Wert auf eine Erholungsstätte $\left(\chi^{2}\right.$-Test $p<0,000$ ). Auch bei anderen Angeboten sind Seniorenhaushalte mit Ausnahme des Bürgertreffs weniger interessiert 
Tab. 1 Veränderungsabsichten. (Datenquelle: Eigene Datenerhebung MAGGIE Haushaltsbefragung 2019)

\begin{tabular}{lllll}
\hline Veränderungsabsichten & $\begin{array}{l}\text { Gesamt } \\
(\%)\end{array}$ & $\begin{array}{l}\text { Nichtseniorenhaushalte } \\
(\%)\end{array}$ & $\begin{array}{l}\text { Seniorenhaushalte } \\
(\%)\end{array}$ & $N$ \\
\hline Auszug von Haushaltsmitgliedern absehbar & 8,0 & 11,8 & 1,5 & 176 \\
Vergrößerung der Wohnsituation & 14,7 & 23,7 & 0,8 & 184 \\
Verkleinerung der Wohnsituation & 6,6 & 6,3 & 7,2 & 181 \\
Umzug sanierte Wohnung & 26,5 & 35,7 & 11,4 & 185 \\
Umzug barrierefreie Wohnung & 17,8 & 18,6 & 16,4 & 180 \\
\hline
\end{tabular}

Tab. 2 Wünsche für soziale Treffpunkte. (Datenquelle: Eigene Datenerhebung MAGGIE Haushaltsbefragung 2019, Mehrfachnennung möglich)

\begin{tabular}{lllll}
\hline Soziale Treffpunkte & $\begin{array}{l}\text { Gesamt } \\
(\%)\end{array}$ & $\begin{array}{l}\text { Nichtseniorenhaushalte } \\
(\%)\end{array}$ & $\begin{array}{l}\text { Seniorenhaushalte } \\
(\%)\end{array}$ & $\begin{array}{l}N \\
\text { Quartiersladen }\end{array}$ \\
Erholungsstätte wie Außensitzplatz & 45,6 & 50,8 & 37,0 & 195 \\
Pflegedienstanbieter & 40,0 & 50,8 & 21,9 & 195 \\
Begegnungsstätte wie Bürgertreff & 26,7 & 27,9 & 24,7 & 195 \\
Freizeitstätte wie Spielplatz & 23,6 & 23,0 & 24,7 & 13,7 \\
Gemeinschaftsaktivitäten im kulturellen, sozialen Bereich & 20,5 & 24,6 & 12,3 & 195 \\
usw. & 13,8 & 14,8 & & \\
\hline
\end{tabular}

Tab. 3 Elemente des Sozialkapitalindex. (Datenquelle: Eigene Datenerhebung MAGGIE Haushaltsbefragung 2019)

\begin{tabular}{|c|c|}
\hline Faktoren des Sozialkapitalindexes & Skalenart \\
\hline Zusammenlebend in Ehe & Binär (dichotome Auswahl): ja, nein \\
\hline Verwandtschaft in der Margaretenau & Binär (dichotome Auswahl): ja, nein \\
\hline $\begin{array}{l}\text { Hohe Zufriedenheitswerte mit Zusam- } \\
\text { menleben im Wohnblock }\end{array}$ & 4-stufige Skala dichotom kodiert: sehr zufrieden, zufrieden, weniger zufrieden, sehr unzufrieden \\
\hline Hohe Kontaktintensität mit Nachbarschaft & $\begin{array}{l}\text { 4-stufige Skala dichotom kodiert: sehr häufig (wöchentlich), ab und zu (monatlich)/selten (einige } \\
\text { Male jährlich), überhaupt nicht }\end{array}$ \\
\hline $\begin{array}{l}\text { Ehrenamtsquote (freiwillige ehrenamtli- } \\
\text { che Tätigkeit) }\end{array}$ & Binär (dichotome Auswahl): ja/nein \\
\hline $\begin{array}{l}\text { Engagementbereitschaft für die Genos- } \\
\text { senschaft }\end{array}$ & Binär (dichotome Auswahl): ja/nein \\
\hline Häufig geleistete Nachbarschaftshilfe & $\begin{array}{l}\text { 4-stufige Skala dichotom kodiert: sehr häufig (wöchentlich), ab und zu (monatlich), selten (einige } \\
\text { Male jährlich), überhaupt nicht }\end{array}$ \\
\hline Häufig erhaltene Nachbarschaftshilfe & $\begin{array}{l}\text { 4-stufige Skala dichotom kodiert: sehr häufig (wöchentlich), ab und zu (monatlich), selten (einige } \\
\text { Male jährlich), überhaupt nicht }\end{array}$ \\
\hline
\end{tabular}

als jüngere Haushalte, wobei die Unterschiede nicht signifikant sind. Bei dem Wunsch jüngerer Haushalte nach einem ambulanten Pflegedienst im Quartier denken sie vermutlich an ihre älteren Angehörigen.

\section{Soziales Kapital}

Der Begriff soziales Kapital wurde 1961 in der stadtsoziologischen Studie von Jane Jacobs eingebracht, in der sie verschiedene Funktionen von Nachbarschaftsvierteln und den darin enthaltenen städtischen Anlagen untersuchte (Haug und Gerlitz 2007). Jacobs führt positive Aspekte des sozialen Kapitals vor allem auf die Stadtplanung, Straßenführung und Architektur in Wohngebieten zurück, die das Gemeinschaftsgefühl und die Entstehung von Be- ziehungsnetzen entscheidend beeinflussen können (1961). Nach Schnur (2010) können besonders in stark vom Alter geprägten Quartieren nachbarschaftsorientierte, intergenerationelle Beziehungen stabilisierend wirken. Die Berechnung des lokalen Sozialkapitalindexes erfolgt nach Schnur (2003) als additiver Index (Tab. 3).

Der so berechnete Sozialkapitalindex variiert zwischen 0,13 und 0,88 , wobei er folgend interpretiert werden kann: 0,13 bedeutet kaum Sozialkapital und 0,88 sehr starkes soziales Kapital. Die Quartiersbevölkerung weist relativ hohes Sozialkapital auf (Median 0,63). Dieser ist bei den Seniorenhaushalten niedriger als bei den jüngeren Haushalten (Abb. 5). Auch der Mittelwert ist bei Seniorenhaushalten $(N=59)$ mit 0,5 (SD 0,17) niedriger als bei Nichtseniorenhaushalten $(N=102, \mathrm{M} 0,54, \mathrm{SD} 0,19)$. Der Unterschied ist jedoch nicht signifikant. 


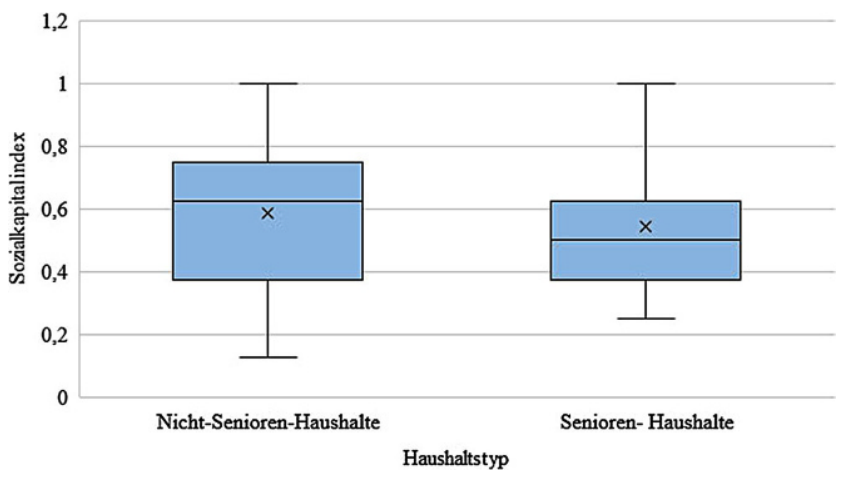

Abb. 5 Sozialkapitalindex. (Datenquelle: Eigene Datenerhebung MAGGIE Haushaltsbefragung 2019, Boxplot mit SPSS 24)

\section{Fazit}

Es konnte belegt werden, dass die genossenschaftliche Wohnform relativ hohe Wohnzufriedenheit und hohes lokales Sozialkapital nach Schnur (2003) erzeugt. Das in der Literatur beschriebene Konzept ageing in place (Davey et al. 2004) konnte bestätigt werden. Der Anteil der nichtumzugsbereiten Senioren-Haushalte übertraf andere Studien (Teti et al. 2014). Das geringere Interesse der befragten Seniorenhaushalte an barrierereduziertem Wohnen könnte damit zusammenhängen, dass es ihr größter Wunsch ist, die eigene Wohnung und direkte Nachbarschaft nicht zu verlassen. Sie bevorzugen eine (energetische) Sanierung im bewohnten Zustand.

Als Implikation für die Stadtentwicklungsplanung kann abgeleitet werden, dass bei Konzepten für die Gebäude- und Quartierssanierung eine Analyse der Altersstruktur und eine Bedarfserhebung eine hilfreiche Grundlage darstellen. In stärker von demografischer Alterung betroffenen Quartieren sollten die besonderen Bedürfnisse von Seniorenhaushalten in den Blick genommen werden. Gute Nahversorgung, altersgerechte Begegnungsstätten und Pflegedienstanbieter sind hierbei ein Beispiel. Diese Ergebnisse werden als Empfehlung Eingang in das Konzept des Sanierungsmanagements der Margaretenau in Regensburg finden.

Danksagung Das Projekt Energetische Modernisierung des genossenschaftlichen Wohnquartiers Margaretenau in Regensburg (MAGGIE) der Ostbayerischen Technischen Hochschule (OTH) Regensburg wird gefördert durch das Bundesministerium für Wirtschaft und Energie (Förderlinie Solares Bauen, Kennzeichen 03SBE0007). Mit dem Sanierungsmanagement Margaretenau wurde das Architekturbüro Luxgreen beauftragt (gefördert aus KfW-Bundesmitteln und von der Stadt Regensburg 2018).

Funding Open Access funding enabled and organized by Projekt DEAL.

Open Access Dieser Artikel wird unter der Creative Commons Namensnennung 4.0 International Lizenz veröffentlicht, welche die Nutzung, Vervielfältigung, Bearbeitung, Verbreitung und Wiedergabe in jeglichem Medium und Format erlaubt, sofern Sie den/die ursprünglichen Autor(en) und die Quelle ordnungsgemäß nennen, einen Link zur Creative Commons Lizenz beifügen und angeben, ob Änderungen vorgenommen wurden.

Die in diesem Artikel enthaltenen Bilder und sonstiges Drittmaterial unterliegen ebenfalls der genannten Creative Commons Lizenz, sofern sich aus der Abbildungslegende nichts anderes ergibt. Sofern das betreffende Material nicht unter der genannten Creative Commons Lizenz steht und die betreffende Handlung nicht nach gesetzlichen Vorschriften erlaubt ist, ist für die oben aufgeführten Weiterverwendungen des Materials die Einwilligung des jeweiligen Rechteinhabers einzuholen.

Weitere Details zur Lizenz entnehmen Sie bitte der Lizenzinformation auf http://creativecommons.org/licenses/by/4.0/deed.de.

\section{Literatur}

Baugenossenschaft Margaretenau (2018) 100 Jahre Baugenossenschaft Margaretenau 1918-2018. Pustet, Regensburg

Bundesministerium für Verkehr, Bau und Stadtentwicklung (2011) Wohnen im Alter. Marktprozesse und wohnungspolitischer Handlungsbedarf. https://www.bbsr.bund.de/BBSR/DE/veroeffentlich ungen/ministerien/bmvbs/forschungen/2011/Heft147_DL.pdf. Zugegriffen: 22. Juli 2020

Davey J, Nana G, de Joux V, Arcus M (2004) Accommodation options for older people in Aotearoa/New Zealand. NZ Institute for Research on Ageing/ Business and Economic Research Ltd, for Centre for Housing Research. https://thehub.sia.govt.nz/ assets/documents/accommodation_options_for_older_people_ in_aotaotea_new_zealand.pdf. Zugegriffen: 12. März 2020

Eichler A, Holz G (2014) Inklusive Gesellschaft - Teilhabe in Deutschland. Älter werden im Quartier - Sozialraumorientierte Unterstützung älterer Menschen. AWO Bundesverband. https://www.awo. org/sites/default/files/2017-01/AWO_ISS_Inklusion_F4.pdf. Zugegriffen: 4. März 2020

Gädker J, Sinning H, Thalheim K (2012) 50plus als Zielgruppe der Wohnungswirtschaft und Stadtentwicklung. Systematisierungsansätze, Anforderungen und Handlungsstrategien. Institut für Stadtforschung, Planung und Kommunikation. https://www. db-thueringen.de/servlets/MCRFileNodeServlet/dbt_derivate_ 00037520/Band_4_ISP_Schriftenreihe_50plus\%20A.pdf. Zugegriffen: 28. Juli 2020

Haug S, Gerlitz J (2007) Messkonzepte sozialen Kapitals. Eine Betrachtung vor dem Hintergrund der Nachhaltigkeitsdebatte. In: Meyerhoff J, Schwarze R (Hrsg) Jahrbuch Ökologische Ökonomik: Soziale Nachhaltigkeit. Metropolis, Marburg, S 189-218

Haug S, Vernim M (2016) Sozialstudie. In: Steffens O et al (Hrsg) RENARHIS: Nachhaltige energetische Modernisierung und Restaurierung historisch wertvoller Stadtquartiere. Bundesinstitut für Bau-, Stadt- und Raumforschung, Bonn, S 47-73

Jacobs J (1961) The death and life of great American cities. Random House, New York

KfW Bankengruppe (2014) Evaluation des KfW-Programms Altersgerecht Umbauen. Endbericht. https://www.kfw.de/PDF/ Download-Center/Konzernthemen/Research/PDF-Dokumentealle-Evaluationen/Prognos_Evaluation-KfW-Programm-Alters gerecht-Umbauen.pdf. Zugegriffen: 28. Juli 2020

Kremer-Preiß U (2013) Teil 2: Kommunale Quartiersentwicklung in der Praxis. In: Michell-Auli P, Kremer-Preiß U (Hrsg) Quartiersentwicklung. KDA-Ansatz und kommunale Praxis. Kuratorium Deutsche Altershilfe, Köln, S 58-117

Opitz S, Pfaffenbach C (2018) Lebensqualität im ländlichen Raum. Wie bewerten Bewohner ihr Wohnumfeld? Standort 42:171-177. https://doi.org/10.1007/s00548-018-0544-y 
Saup W (1993) Alter und Umwelt. Eine Einführung in die ökologische Gerontologie. Kohlhammer, Stuttgart

Schnur O (2003) Lokales Sozialkapital für die „,soziale Stadt“. Politische Geographien sozialer Quartiersentwicklung am Beispiel Berlin-Moabit. VS, Wiesbaden

Schnur O (2010) Demographischer Impact in städtischen Wohnquartieren. Entwicklungsszenarien und Handlungsoptionen. VS, Wiesbaden

Stadt Regensburg, Amt für Stadtentwicklung (2018) Mietspiegel 2018. Wohnen in Regensburg. https://www.regensburg.de/fm/RBG_ INTER1S_VM.a.253.de/r_upload/mietspiegel-2018-2019-stand24012018.pdf. Zugegriffen: 25. Juli 2020

Stadt Regensburg, Amt für Stadtentwicklung (2019) Statistisches Jahrbuch 2019. http://www.statistik.regensburg.de/publikationen/ jahrbuch.php. Zugegriffen: 24. Sept. 2020

Teti A, Grittner U, Kuhlmey A, Blüher S (2014) Wohnmobilität im Alter. Altersgerechtes Wohnen als primärpräventive Strategie. Z Gerontol Geriat 47:320-328. https://doi.org/10.1007/s00391013-0538-0

Theurl T, Wicher J, Cappenberg C (2013) Eigenschaften und Einstellungen von Bewohnern von Wohnungsgenossenschaften. Arbeitspapiere des Instituts für Genossenschaftswesen der Westfälischen Wilhelms-Universität Münster. https://www.econstor.eu/
bitstream/10419/71277/1/74003815X.pdf. Zugegriffen: 25. Juli 2020

Prof. Dr. Sonja Haug ist Soziologin und seit 2010 Professorin für Empirische Sozialforschung an der Ostbayerischen Technischen Hochschule (OTH) Regensburg. Gemeinsam mit Prof. Dr. Weber leitet sie das Institut für Sozialforschung und Technikfolgenabschätzung (IST). Ihre inhaltlichen Schwerpunkte umfassen Demografie, Familie und Migration sowie soziale Aspekte von Technik in den Bereichen Energie, Mobilität und Gesundheit. Sie leitet die Sozialstudie zum Projekt MAGGIE

Miriam Vetter hat Soziale Arbeit an der OTH Regensburg studiert. Im Anschluss absolvierte sie den Masterstudiengang der Sozialen Arbeit mit dem Schwerpunkt Inklusion und Exklusion. Seit 2019 ist sie wissenschaftliche Mitarbeiterin im Institut für Sozialforschung und Technikfolgenabschätzung (IST) an der OTH Regensburg. Sie bearbeitet die Sozialstudie zum Projekt MAGGIE. Ihre Schwerpunkte sind soziale Aspekte der Gebäudesanierung und Altersforschung. 